\title{
Awareness of central luminance edge is crucial for the Craik-O'Brien-Cornsweet effect
}

\author{
Ayako Masuda ${ }^{*}$, Junji Watanabe ${ }^{2}$, Masahiko Terao ${ }^{2,3}$, Masataka Watanabe ${ }^{4}$, Akihiro Yagi ${ }^{1}$ and \\ Kazushi Maruya ${ }^{2}$
}

${ }^{1}$ Department of Integrated Psychological Science, Kwansei Gakuin University, Nishinomiya, Japan

${ }^{2}$ NTT Communication Science Laboratories, Nippon Telegraph and Telephone Corporation, Atsugi, Japan

${ }^{3}$ Department of Life Sciences, University of Tokyo, Meguro, Japan

${ }^{4}$ Graduate School of Engineering, University of Tokyo, Bunkyo, Japan

\section{Edited by:}

Theofanis Panagiotaropoulos, Max

Planck Institute for Biological

Cybernetics, Germany

\section{Reviewed by:}

Huseyin Boyaci, Bilkent University, Turkey

Dale Purves, Duke-NUS Graduate

Medical School, Singapore

Claudia Lunghi, Università degli Studi

di Firenze, Italy

\section{*Correspondence:}

Ayako Masuda, Motorcycle R\&D

Center, Honda R\&D Co., Ltd., 3-15-1

Senzui, Asaka-shi, Saitama 351-8555, Japan.

e-mail:ayako.masuda@mail.a.rd.

honda.co.jp
The Craik-O'Brien-Cornsweet (COC) effect demonstrates that perceived lightness depends not only on the retinal input at corresponding visual areas but also on distal retinal inputs. In the $\mathrm{COC}$ effect, the central edge of an opposing pair of luminance gradients (COC edge) makes adjoining regions with identical luminance appear to be different. To investigate the underlying mechanisms of the effect, we examined whether the subjective awareness of the $\mathrm{COC}$ edge is necessary for the generation of the effect. We manipulated the visibility of the COC edge using visual backward masking and continuous flash suppression while monitoring subjective reports regarding online percepts and aftereffects of adaptation. Psychophysical results showed that the online percept of the COC effect nearly vanishes in conditions where the $\mathrm{COC}$ edge is rendered invisible. On the other hand, the results of adaptation experiments showed that the COC edge is still processed at the early stage even under the perceptual suppression. These results suggest that processing of the COC edge at the early stage is not sufficient for generating the $\mathrm{COC}$ effect, and that subjective awareness of the COC edge is necessary.

Keywords: Craik-O'Brien-Cornsweet effect, lightness perception, visual awareness, visual masking, binocular suppression, continuous flash suppression

\section{INTRODUCTION}

The perception of lightness is a fundamental aspect of vision, and it depends not only on the retinal input at the corresponding visual area but also on the distal retinal inputs (e.g., Gilchrist, 1977; Adelson, 1993). The Craik-O'Brien-Cornsweet (COC) effect (O'Brien, 1958; Craik, 1966; Cornsweet, 1970) has been studied to provide a clue as to the underlying mechanisms of lightness processing in the brain. In the COC effect, a central edge of an opposing pair of luminance gradients (COC edge) makes adjoining regions with identical luminance appear to be different. Recent brain imaging and physiological studies have shown that when the COC effect is observed, the early visual cortical areas, starting as early as the primary visual cortex (V1 or V2), are activated (e.g., Roe et al., 2005; Boyaci et al., 2007; Hung et al., 2007; for reviews von der Heydt et al., 2003; Komatsu, 2006). Furthermore, there is evidence suggesting that activity of higher cortical area, such as the lateral occipital (LO) sulcus, is correlated to the illusory lightness perception (Perna et al., 2005). On the other hand, regarding psychophysical studies, although it was suggested that the COC effect is influenced by higher-stage processing of planar surface attributes (Knill and Kersten, 1991), most of the studies have been performed only by modulating the physical features of the COC edge, such as luminance contrast, spatial frequency, and direction, which were assumed to be processed at the early stage of human visual system (Grossberg and Todorovic, 1988; Paradiso and Nakayama, 1991; Davey et al., 1998; Devinck et al., 2007; Perna and Morrone, 2007).
Meanwhile involvement of subjective awareness, whose processing presumably includes the higher stages (for a review Rees et al., 2002), has not been studied directly. In this work, consequently, we examined whether the COC effect can be observed when subjective awareness of the COC edge is suppressed.

We used visual backward masking (BM; Breitmeyer and Ganz, 1976; Breitmeyer and Ogmen, 2000) and continuous flash suppression (CFS; Tsuchiya and Koch, 2005) to manipulate the visibility of the COC edge. In the BM experiment, the masking stimulus was presented immediately after the COC stimulus, which leads to failure to consciously perceive the corresponding area. The masking stimulus was presented in the area of the COC edge to selectively eliminate the visibility of the edge. In the CFS experiment, observer's visual percept was continuously suppressed by presenting a dynamic Mondrian stimulus and the COC stimulus to the dominant eye and the other eye, respectively. CFS could selectively render the COC edge invisible for a few $10 \mathrm{~s}$ in all trials. Our results demonstrate that the COC effect almost completely vanished in both conditions (BM and CFS) when the COC edge was invisible (The results in CFS experiment agree with the observation in Supplemental Data of Boyaci et al., 2007).

The BM and CFS experiments phenomenologically showed that when the subjective percept of the COC edge was suppressed, the lightness induction of the COC effect was not observed, but the visual processing associated with the subjective awareness is still unclear. If the neural processing at the early stage did not 
survive under BM and CFS conditions, loss of subjective awareness could not be regarded as the prime determinant of inhibition of lightness induction in those experiments. In the next experiment (adaptation experiment), we examined whether the COC edge was still processed at the early stage of the visual system, when the COC edge was rendered invisible. In the adaptation experiment, we examined the luminance aftereffect of the COC edge presented under the CFS condition, since the duration of perceptual suppression produced by the CFS enabled us to use the adaptation paradigm to assess the neural processing psychophysically. The results of adaptation experiments showed that the COC edge is still processed at the early stage even under the perceptual suppression of the COC edge. Together with the BM and CFS experiments, it can be suggested that the early-stage processing of the COC edge itself is not sufficient for generating the COC effect, and that subjective awareness of the COC edge is crucial.

\section{MATERIALS AND METHODS OBSERVERS}

Five observers participated in all experiments, and the observers in each experiment included one or two of the authors. The rest of the observers were volunteers unaware of the purpose of the experiments. All observers had normal or corrected-to-normal vision. The dominant eye was determined for each observer by the Dolman method (Fink, 1938). Informed consent was obtained from the naïve participants before the experiment started. Recruitment of the participants and experimental procedures were conducted in accordance with the Declaration of Helsinki.

\section{APPARATUS}

Stimuli were generated with a PC/AT compatible personal computer using the Psychlops library (Maruya et al., 2010), and displayed on a 21 -inch CRT monitor (TOTOKU Calix CDT2141A), with a refresh rate of $100 \mathrm{~Hz}$. An 8-bit grayscale with gamma correction was provided by a video card (Aopen GeForce4Ti4200 with AGP8X). The observer viewed the monitor from a distance of $64 \mathrm{~cm}$ while sitting in a completely dark room with his/her head fixed on a chin rest. The spatial resolution of the monitor was $1280 \times 1024$ pixels, with each pixel subtending 1.6 arc minutes at the viewing distance of $64 \mathrm{~cm}$. In the BM experiment, visual stimulus was presented at the center of the monitor, and the observers viewed the stimuli with two eyes. In the CFS and adaptation experiments, the display area of the monitor was horizontally divided into two areas and the observers viewed two stimuli presented in each area through a mirror stereoscope so that each eye could see its corresponding stimuli.

\section{VISUAL STIMULI}

Figure 1A shows the COC stimulus used in the BM and CFS experiments. The width and height of the stimulus were 9.4 and 5.3 arc degree, respectively. The size of the luminance gradient region was $2.4 \times 5.3$ arc degree. The observers compared the perceived lightness of left and right flanking regions. As shown in Figure 1B, the mean luminance of the flanking region was $24.0 \mathrm{~cd} / \mathrm{m}^{2}$. When the left and right flanking regions are physically equiluminant, they appear to be different in lightness due to the presence of the COC edge. The maximum and minimum luminances of the COC edge were 27.6 and $19.9 \mathrm{~cd} / \mathrm{m}^{2}$, respectively.
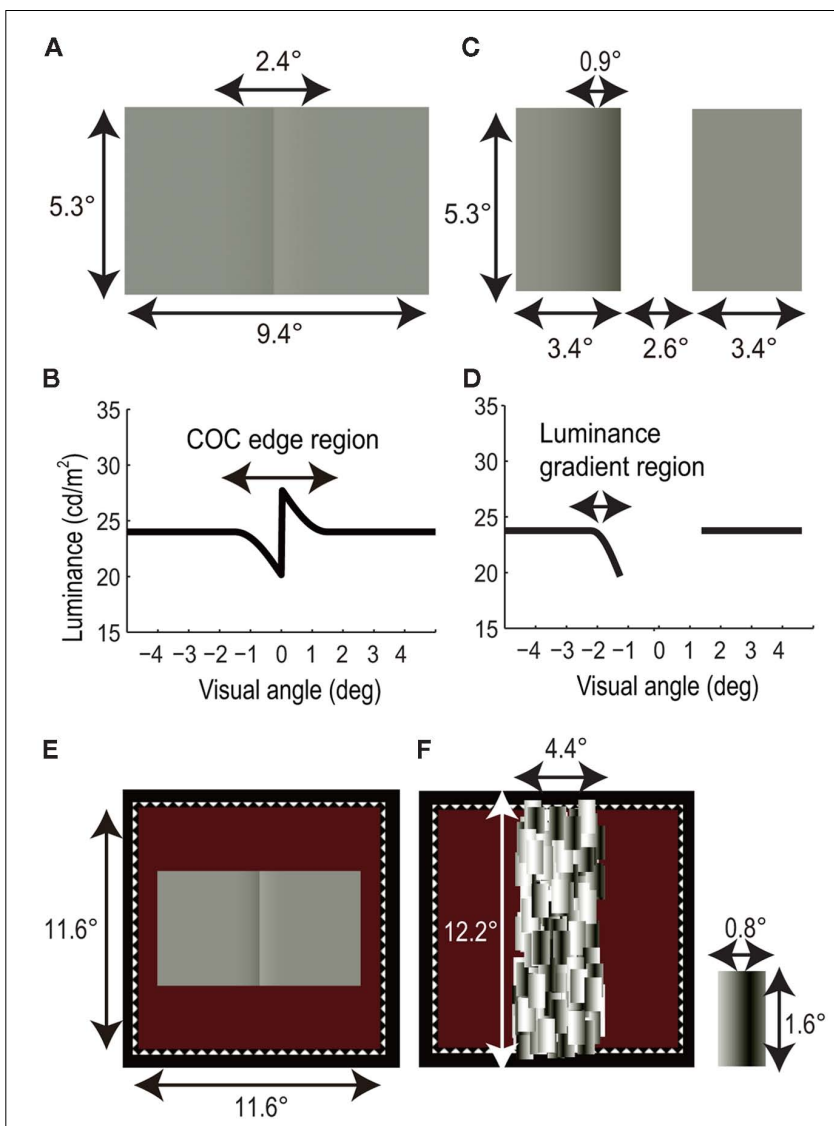

FIGURE 1 | Visual stimuli. (A) Craik-O'Brien-Cornsweet (COC) stimulus. (B) Luminance profile of COC stimulus. (C) Modified COC stimulus consists of two rectangles. (D) Luminance profile of modified COC stimulus. (E) COC stimulus with white dotted line and red background. (F) Mondrian stimulus and a rectangular patch of Mondrian stimulus.

Figure 1C shows a modified COC stimulus used in the adaptation experiment. The modified COC stimulus was composed of two rectangles $3.4 \times 5.3$ arc degree in size, arranged with 2.6 arc degree horizontal spatial interval. One of the rectangles had a luminance gradient $0.9 \times 5.3$ arc degree in size, which caused lightness induction into the whole rectangle area. The baseline and minimum luminances of the luminance gradient were 24.0 and $19.9 \mathrm{~cd} / \mathrm{m}^{2}$, respectively (Figure 1D). We used the modified COC stimuli instead of the original COC stimulus, since this enabled us to measure the aftereffect of the COC edge without influence on its lightness induction into adjacent areas.

As shown in Figure 1E, the COC stimulus and the modified COC stimulus were presented with a square frame $(11.6 \times 11.6$ arc degree) drawn with white dotted lines ("fuse frame"). The background color was dark red $\left(0.11 \mathrm{~cd} / \mathrm{m}^{2},(\mathrm{CIE} 1931) ; x=0.476\right.$, $y=0.523)$ in the BM and CFS experiments, and it was red $\left(3.73 \mathrm{~cd} / \mathrm{m}^{2},(\mathrm{CIE} 1931) ; x=0.494, y=0.375\right)$ in the adaptation experiment to optimize the adaptation effect (These values were decided on the basis of preliminary observations). Before the initiation of a trial in all experiments, fixation targets (white crosses $0.53 \times 0.53$ arc degree in size and $78 \mathrm{~cd} / \mathrm{m}^{2}$ in luminance) were presented for stable fixation at the center of the stimulus and 6.3 
arc degree above and below it. The three fixation targets were presented to avoid misalignments caused by ocular torsion.

In the BM experiment, a static Mondrian stimulus was presented immediately after the COC stimulus. As shown in Figure 1F, the size of the Mondrian stimulus was $4.4 \times 12.2$ arc degree. The Mondrian stimulus was composed of patches of rectangles, subtended $0.8 \times 1.6$ arc degree and consisting of horizontal sinusoidal gratings. The spatial frequency of the sinusoidal gratings was 0.63 cycle/degree. The COC and masking stimuli were presented to both eyes. The central positions of the COC and Mondrian stimuli were aligned in the displayed area, and the area of the Mondrian stimulus totally covered the COC edge.

In the CFS experiment, a dynamic Mondrian stimulus and the COC stimulus were simultaneously presented to the dominant eye and the other eye, respectively. We used a dynamic Mondrian stimulus composed of drifting sinusoidal gratings instead of static rectangles, since it allowed us to render the COC edge invisible completely and continuously for several tens of seconds (Maruya et al., 2008). The size and position of the Mondrian stimulus were the same as those in the BM experiment. The rectangles of the Mondrian stimulus comprised dynamic horizontal sinusoidal gratings, which were individually moved horizontally at the speed of $5.33 \%$ s. The direction of motion (left or right) was reversed with random timing to avoid motion adaptation by these gratings. The arrangement of these rectangles changed every $500 \mathrm{~ms}$. The central positions of the COC and dynamic Mondrian stimuli were aligned. The Mondrian stimulus totally covered the COC edge during stimulus presentation and interocularly suppressed the awareness around the COC edge ${ }^{1}$.

In the adaptation experiment, luminance aftereffects of the invisible COC edge were tested. In the adaptation phase, the dynamic Mondrian stimulus used in the CFS experiment and a modified COC stimulus were simultaneously presented to the dominant eye and the other eye, respectively. The positions of the Mondrian stimulus and modified COC stimulus were aligned. The Mondrian stimulus totally covered the luminance gradient region of one rectangle and interocularly suppressed the awareness around the luminance gradient region ${ }^{1}$. In the test phase, two uniform rectangles with $24.0 \mathrm{~cd} / \mathrm{m}^{2}$ of luminance were presented to the dominant eye or the other eye. The sizes, positions, and eye of the test rectangles were changed depending on the experimental conditions. In the standard test condition, the test rectangles were the same as the modified COC stimulus in size and position; in the flanking test condition, the test rectangles $1.8 \times 5.3$ arc degree in size arranged with a 5.8 arc degree horizontal spatial interval were used.

\section{BM EXPERIMENT \\ Procedure}

In the mask condition (Figure 2A), a COC stimulus was presented for $50 \mathrm{~ms}$, and a Mondrian stimulus was presented for $10 \mathrm{~ms}$

\footnotetext{
${ }^{1}$ It is known that interocular suppression is not strictly confined to the retinal area of rival stimulation, but spreads beyond the boundaries of those stimuli (e.g., Kaufman, 1963; Blake and Camisa, 1979; Liu and Schor, 1994). The spatial extent of suppression can spread across several degrees when the suppression is strong (Maruya and Blake, 2009). However, in this study, we adjusted the strength of suppression so that the suppression did not reach the area the observers used to judge the perceived lightness.
}

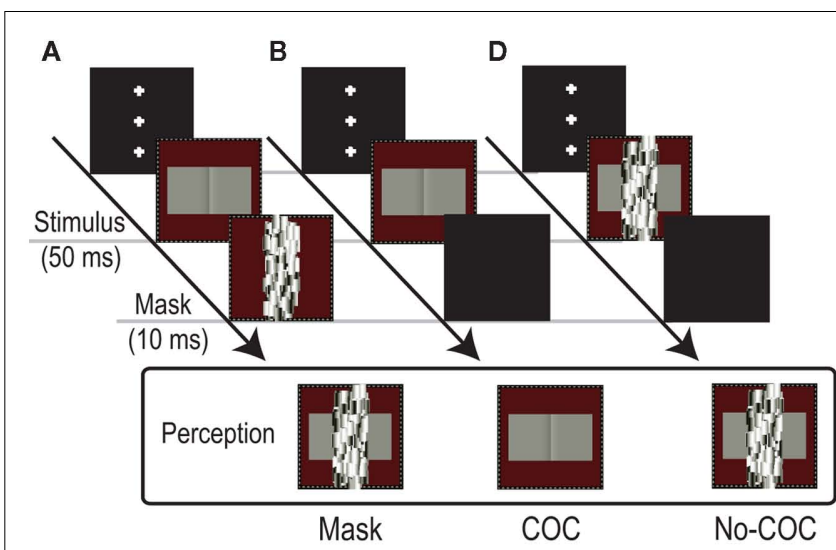

D
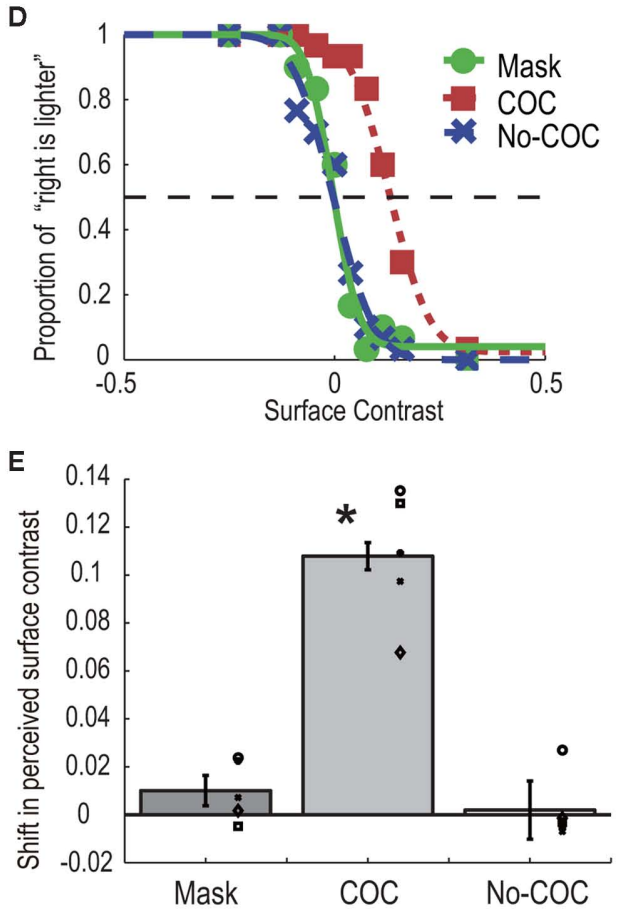

FIGURE 2 | Schematic illustrations of procedure and visual stimuli in the BM experiment: (A) Mask condition, (B) COC condition, and (C) No-COC condition. (D) Psychometric functions obtained with observer AM. (E) Shifts of PSEs in the three conditions. The bars indicate the averaged shift of PSE, with error bars showing \pm SEs. Symbols indicate individual data points.

immediately after the COC stimulus disappeared. Observers were asked to make a two-alternative forced choice about which surface region of the COC stimulus was perceived lighter (left or right). Prior to the experiment we confirmed that all observers could not perceive the COC edge in this condition. The luminance of left and right flanking regions were systematically changed from trial-to-trial, while the polarity of COC edge was fixed so that the right flanking region was perceived lighter when the flanking regions of both sides had the same luminance. The Michelson contrast of the flanking surface regions of the COC stimulus was varied in 10 steps: $-0.24,-0.12,-0.08,-0.04,0,0.04,0.08,0.12$, 0.16 , and 0.32 . Negative values mean that luminance of the right flanking region was high and vice versa. At the lowest contrast 
-0.24 , the luminances of left and right flanking regions were 18.2 and $29.7 \mathrm{~cd} / \mathrm{m}^{2}$, respectively. At the highest contrast 0.32 , the luminances of the left and right flanking regions were 31.4 and $16.1 \mathrm{~cd} / \mathrm{m}^{2}$, respectively. For the purpose of control, we performed experiments in which the Mondrian stimulus was not presented (COC condition, see Figure 2B) and in which the Mondrian stimulus superimposed on the COC stimulus was presented for $50 \mathrm{~ms}$ (No-COC condition, see Figure 2C). Thirty trials were performed for each contrast value (300 trials for one condition, 900 trials in total). Trials in the three conditions were performed in a randomized order.

\section{Results}

Figure 2D shows psychometric functions of an observer (AM) under the three conditions. In general, when the surface contrast was negative (the luminance of right region was high), the observers reported that they perceived the right region as being lighter, indicating that the observers could judge the surface lightness properly. Although both psychometric functions obtained in the Mask and No-COC conditions were non-biased, that in the COC condition was shifted in the direction of positive surface contrast (the luminance of left region was high). The apparent lightness was estimated for each condition by calculating the point of subjective equality (PSE) with the cumulative Gaussian function fitted to the psychometric function. The averaged PSEs across all observers with SEs are shown in Figure 2E. A positive value means that a typical COC effect occurred. A significant shift toward positive surface contrast from zero was observed in the COC condition $[t(4)=8.87, p<0.05]$, indicating that the COC effect could occur even for very short stimulus duration. Conversely, no shift in the PSE was observed in the Mask condition $[t(4)=1.78$, $p=0.15]$ and No-COC condition $[t(4)=0.31, p=0.77]$. A oneway repeated measures ANOVA revealed that there was a significant difference between conditions $[F(2,8)=74.48, p<0.01]$. The post hoc comparison (Ryan's method, significant level $=0.05$ ) showed that the value in the COC condition was significantly higher than those in the Mask and No-COC condition, and that there was no significant difference between the Mask condition and No-COC conditions. These results demonstrated that when the percept of the COC edge was suppressed by a BM stimulus, the COC effect was reduced to the identical level where the COC edge was physically covered.

\section{CFS EXPERIMENT}

\section{Procedure}

A dynamic Mondrian stimulus and the COC stimulus were presented to the dominant eye and the other eye, respectively. The Mondrian stimulus interocularly suppressed the awareness of the COC edge (CFS condition, see Figure 3A). The observers were asked to make a two-alternative forced choice about which region was perceived lighter as the Michelson contrast of the flanking regions was systematically changed in the same manner as in the BM experiment. When observers detected the COC edge at any time during a trial, they were asked to report it by pressing a button to abort the trial, and the same trial condition was presented afterward. Such aborted trials were rare, occurring only one or two times for the whole experiment at most. For control, we performed

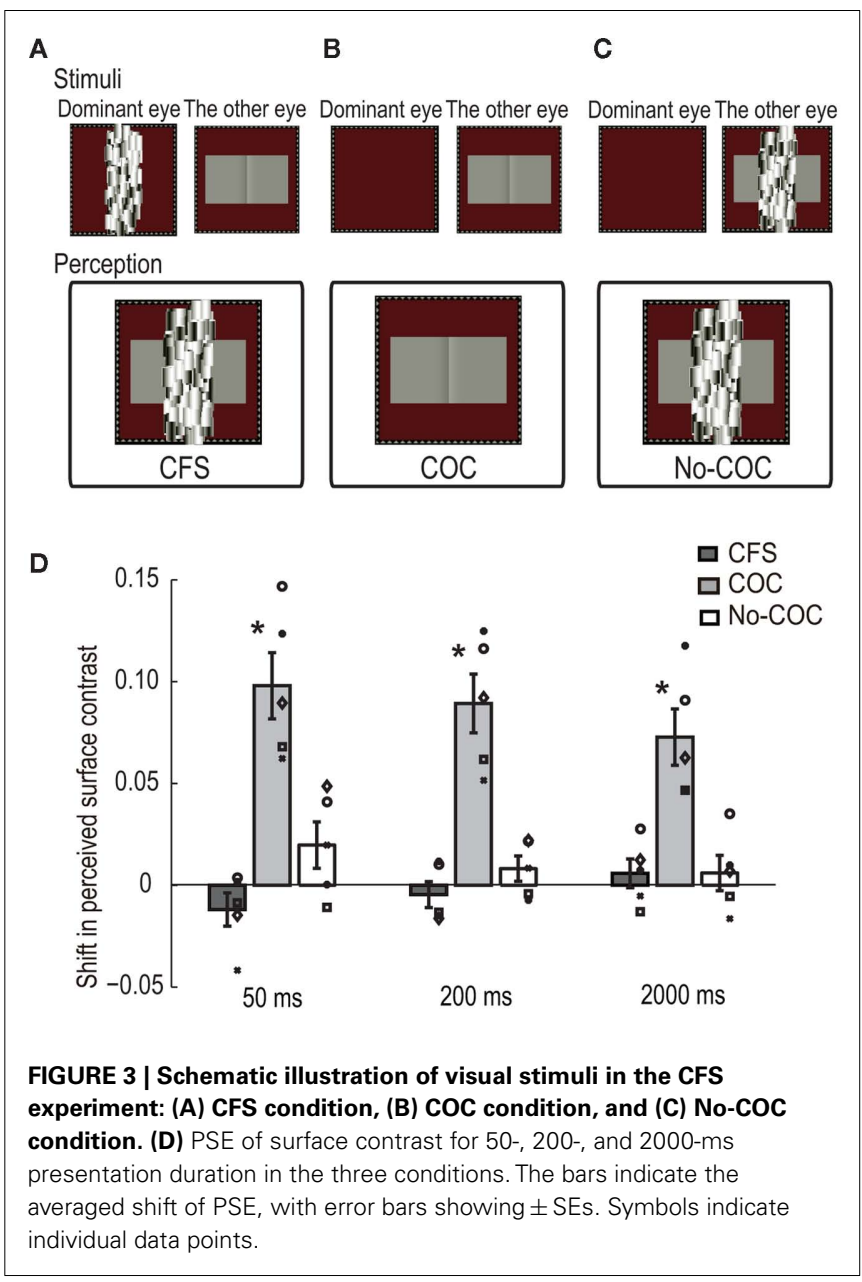

two additional conditions, one in which the dynamic Mondrian stimulus was not presented (COC condition, see Figure 3B) and another in which the Mondrian stimulus superimposed on the COC stimulus was presented to the non-dominant eye (No-COC condition, see Figure 3C). The presentation duration of the COC stimulus was 50,200 , or $2000 \mathrm{~ms}$. Trials with the three durations and three conditions were performed in a randomized order. Thirty trials were performed for each contrast value (900 trials for one condition, 2700 trials in total).

\section{Results}

We estimated PSEs to quantitatively evaluate apparent lightness under the presentation of CFS. Figure 3D shows results for all conditions with the three presentation durations. The vertical axis represents the shift of PSEs in the surface contrast. A positive value means that a typical COC effect occurred. The averaged PSEs across all observers with SEs are shown. The amounts of shift in the COC condition were significantly larger than zero for all durations $[t(4)=6.03, p<0.05$ for $50 \mathrm{~ms}, t(4)=6.18$, $p<0.05$ for $200 \mathrm{~ms}, t(4)=5.27, p<0.05$ for $2000 \mathrm{~ms}$ ], although the magnitude of the COC effect slightly decreased as the presentation duration increased. On the other hand, the amounts of shift were not significantly different from zero in the CFS 
condition $[t(4)=1.46, p=0.22$ for $50 \mathrm{~ms}, t(4)=0.72, p=0.51$ for $200 \mathrm{~ms}, t(4)=0.83, p=0.46$ for $2000 \mathrm{~ms}$ ] or No-COC conditions $[t(4)=1.73, p=0.16$ for $50 \mathrm{~ms}, t(4)=1.31, p=0.26$ for $200 \mathrm{~ms}, t(4)=0.67, p=0.54$ for $2000 \mathrm{~ms}$ ].

In order to evaluate the statistical difference in the shifted PSE between conditions at each presentation duration, we first performed a two-way repeated measures ANOVA with condition and duration as factors, and the shift of PSE as the dependent variable. The results showed a significant difference between the conditions $[F(2,8)=48.86, p<0.01]$ and a non-significant difference between the durations $[F(2,8)=3.40, p=0.09]$. The interaction between them was significant $[F(4,16)=3.98, p<0.05]$. Simple main effects were tested between conditions under each duration, and the results revealed that there were significant differences between the conditions under all durations $[F(2,24)=45.92, p<0.01$ for $50 \mathrm{~ms}, F(2,24)=37.20, p<0.01$ for $200 \mathrm{~ms}, F(2,24)=21.38, p<0.01$ for $2000 \mathrm{~ms}]$. The post hoc multiple comparison (Ryan's method, significant level $=0.05$ ) showed that the values in the COC condition were significantly higher than those in the CFS and No-COC conditions for all durations. These results demonstrated that in conditions where the visual awareness of the COC edge was suppressed by CFS, the COC effect was drastically reduced to the identical level where the COC edge was physically covered.

Simple main effects were also tested between durations for each condition, and a significant difference was only acquired in the COC condition $[F(2,24)=3.14, p=0.06$ for CFS condition, $F(2,24)=6.42, p<0.01$ for COC condition, $F(2,24)=2.14$, $p=0.13$ for No-COC condition]. The post hoc multiple comparisons (Ryan's method, significant level $=0.05$ ) showed that the values for the 50- and 200-ms durations were not significantly different but were significantly higher than for the 2000-ms duration condition. This tendency for the strength of the COC effect to decrease with increasing presentation duration is consistent with the result of a previous study (Wachtler and Wehrhahn, 1997). Although we do not clearly know the reason behind this, it might reflect temporal dynamics of the lightness induction process, which might be composed of a fast process for generating an initial percept of surface lightness (Robinson and de Sa, 2008) and a slow process to fill the lightness information into the whole surface area (De Valois et al., 1986; Rossi and Paradiso, 1996).

\section{ADAPTATION EXPERIMENT}

Both the BM and CFS experiments phenomenologically demonstrated that when the subjective percept of the COC edge was suppressed, the COC effect was not observed. However, the processing stage of this phenomenon is still unclear. The results of the BM and CFS experiments lead to, at least, two possibilities: (i) the neural processing of the COC edge at the early stage was suppressed or (ii) the COC edge was still processed at the early stage, but the subsequent process of lightness induction was suppressed. Our next investigation used an adaptation paradigm to look into the processing stage of the COC edge. Specifically, we measured the luminance aftereffects of the COC stimulus under conditions where the COC edge was rendered invisible during the adaptation period.

\section{Procedure}

In the COC + CFS condition, as shown in Figure 4A, the dynamic Mondrian stimulus and the modified COC stimulus was presented to the dominant eye and the other eye, respectively, for $8000 \mathrm{~ms}$ during the adaptation phase. One rectangle of the modified COC stimulus had a luminance gradient region (gradient rectangle), in which lightness induction into the whole rectangle area was observed, and the other had uniform luminance (see Figure 1C).

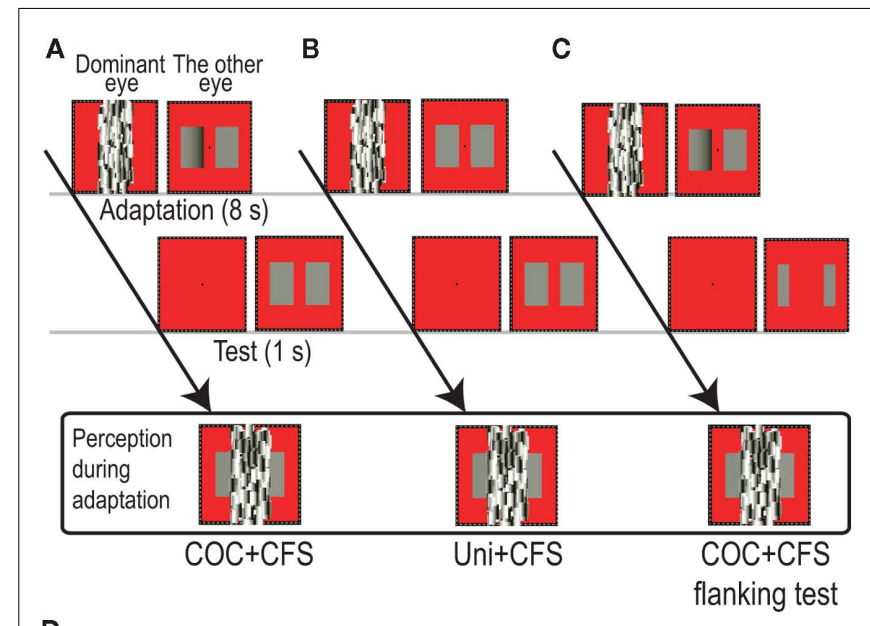

D

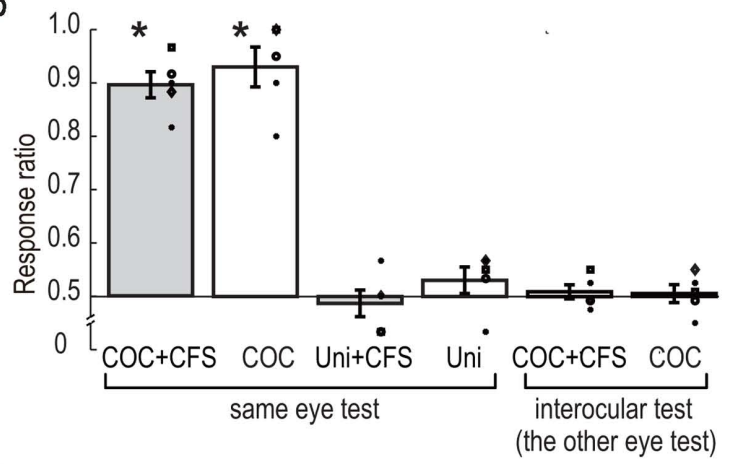

E

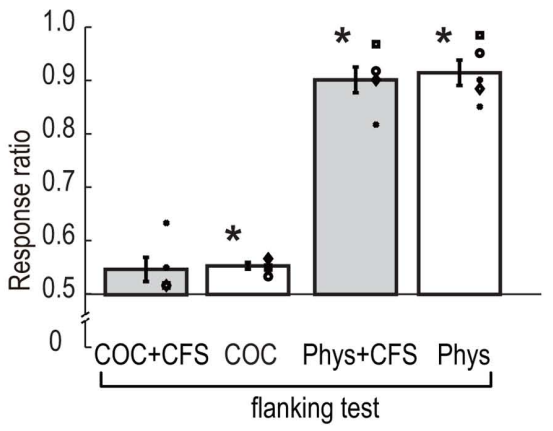

FIGURE 4 | Schematic illustration of procedure and visual stimuli in adaptation experiment: (A) CFS standard test condition, (B) CFS equiluminant adaptation condition, and (C) CFS flanking test condition. (D) Ratio of the rectangle at the side of luminance gradient reported as brighter in the six conditions of the adaptation experiment. The bars indicate the averaged rate, with error bars showing \pm SEs. Symbols indicate individual data points. (E) Ratio of the rectangle at the side of luminance gradient reported as brighter in the four conditions of the subsidiary adaptation experiment. 
In the test phase, two rectangles with uniform luminance (standard test rectangles) were presented to the non-dominant eye for $1000 \mathrm{~ms}$, and observers reported which rectangle they perceived to be lighter (right or left). When observers detected the luminance gradient at any time during adaptation, they were asked to report it by pressing a button to abort the trial, and the same trial condition was presented afterward. As a control experiment, we tested a condition in which both the two rectangles for adaptation had uniform luminance (Uni + CFS condition, see Figure 4B). The luminance of the uniform rectangles was $24.0 \mathrm{~cd} / \mathrm{m}^{2}$, which was the same as the luminance of the flanking region of the modified COC stimulus. As reported in the CFS experiment, observers could not tell which rectangle had the luminance gradient in the COC + CFS condition. Therefore, the perceived stimuli during the adaptation were subjectively the same in the COC + CFS and Uni + CFS conditions.

To check the basic adaptation effects of the modified COC stimulus, we presented the modified COC stimulus or the equiluminant rectangles without the CFS stimulus for adaptation (COC, and Uni conditions). In addition, to examine the stage of adaptation, we also used conditions, in which the standard test rectangles were presented to the opposite eye to which the modified COC had been presented. After adapting to the modified COC stimulus to the non-dominant eye with CFS to the dominant eye (COC + CFS interocular test condition), or without CFS (COC interocular test condition), the standard test rectangles were presented to the dominant eye. The number of trials in which the gradient rectangle was presented at one side (right or left) was set to 30 , and these 30 trials were blocked in the COC + CFS, COC, and the two interocular test conditions ( 60 trials in total for each condition). The number of trials in the Uni + CFS and Uni conditions was set to 30 .

\section{Results}

The average ratios of observers' perceiving a test rectangle lighter at the location where the gradient rectangle was presented are shown in Figure 4D. In the COC + CFS, and COC conditions, the ratios were around $90 \%$, and significantly higher than chance level $[t(4)=11.15, p<0.05$ for COC $+\mathrm{CFS}$, and $t(4)=6.42, p<0.05$ for COC]. On the other hand, the ratios of the Uni + CFS and Uni conditions were not significantly different from chance level $[t(4)=0.53, p=0.62$ for Uni + CFS, and $t(4)=1.20, p=0.30$ for Uni]. We observed no statistically significant effect of interocular transfer both with CFS and without CFS, ratios of subjective report being not significant from chance level $[t(4)=0.63, p=0.56$ for COC + CFS interocular test, and $t(4)=0.30, p=0.78$ for COC interocular test].

To statistically analyze the relationship between the conditions, we performed a two-way repeated measures ANOVA with the type of CFS (with or without CFS) and stimulus combination of adaptation-test (COC-standard test, Uni-standard test, and COCinterocular test) as factors and the ratio as the dependent variable. The results showed non-significant difference for the type of CFS $[F(1,4)=2.46, p=0.19]$ and a significant difference for the type of stimulus combination $[F(2,8)=62.13, p<0.01]$. The interaction between them was not significant $[F(2,8)=2.58, p=0.14]$. These statistical analyses showed that the ratio in the COC + CFS condition was equivalent to that in the COC condition, suggesting that the CFS did not interfere with the processing of luminance adaptation.

The post hoc comparison (Ryan's method, significant level $=0.05$ ) showed that the values in COC-standard test stimulus combination were significantly higher than those for the Uni-standard test and COC-interocular test combination, and that there was no significant difference between the Uni-standard test and COC-interocular test combinations. These analyses indicate that, although the perceptions during adaptation were subjectively the same in the COC + CFS and Uni + CFS conditions, their ratios were significantly different, and this provided evidence that the adaptation effect in the COC + CFS condition was not an artifact due to observers' being able to see the side of the gradient rectangle. These results supported scenario (ii), indicating that the COC edge was still processed under the CFS, presumably at the early stage of monocular processing (see the results of interocular test conditions), and that the CFS stimulus disrupted subsequent process of lightness induction.

\section{SUBSIDIARY ADAPTATION EXPERIMENT}

Given the results of the above adaptation experiments suggesting that the neural processing of the COC edge seems to survive interocular suppression by CFS, we next attempted to test for the subsequent processing of lightness induction into the adjacent surface using a similar paradigm of adaptation. For this purpose, we presented the test stimulus only in retinotopic regions where the adaptation stimulus had no luminance gradient.

\section{Procedure}

The procedures were the same as the standard test conditions in the adaptation experiment except for the size of the test rectangles. The adaptation stimulus was the modified COC stimulus presented to the non-dominant eye with the CFS stimulus presented to the dominant eye (COC + CFS flanking test condition) or only the modified COC stimulus presented to the non-dominant eye (COC flanking test condition). As shown in Figure 4C, small uniform test rectangles, which did not cover the area of the luminance gradient, were presented in the test phase. The size of the test rectangle was chosen to avoid the spread of the adaptation effect of the luminance gradient region (see the details in the "Visual Stimuli" section). As a control, we tested a condition in which the two rectangles for adaptation had uniform but different luminance. The luminance of one rectangle for adaptation was $24.0 \mathrm{~cd} / \mathrm{m}^{2}$, which was the same as that used in the Uni conditions. The luminance of the other rectangle was adjusted from 18.0 to $20.2 \mathrm{~cd} / \mathrm{m}^{2}$ according to the observers so that the rectangle could be perceived as having the same lightness as illusory lightness of the gradient rectangle of the modified COC stimulus (lower luminance rectangle). During the adaptation phase, the two rectangles were presented to the non-dominant eye with or without the CFS stimulus presented to the dominant eye (Phys + CFS flanking test or Phys flanking test condition), and during the test phase, flanking test rectangles were presented. The number of trials in which the gradient rectangle or lower luminance rectangle was presented at one side (right or left) was set to 30 , and these 30 trials were blocked (60 trials in total for each condition). 


\section{Results}

The average ratios of observers' perceiving a test rectangle lighter at the location where the gradient rectangle was presented are shown in Figure 4E. The ratio for the COC + CFS flanking test condition was not significantly different from chance level $[t(4)=2.05$, $p=0.11]$, and that for the COC flanking test condition was slightly higher than chance level and statistically significant $[t(4)=8.52$, $p<0.01]$. The ratios for the Phys + CFS flanking test and Phys flanking test conditions were significantly higher than chance level $[t(4)=11.46, p<0.01$ for Phys + CFS flanking test; $t(4)=10.46$, $p<0.01$ for Phys flanking test]. We performed a two-way repeated measures ANOVA with type of CFS (with or without CFS) and adaptation stimulus (COC adaptation and Physical adaptation) as factors and the ratio as the dependent variable. The results showed a non-significant difference in the CFS $[F(1,4)=2.37, p=0.20]$, demonstrating again that the CFS did not interfere with the processing of luminance adaptation, and a significant difference in the type of adaptation stimulus $[F(1,4)=63.13, p<0.01]$. The interaction between them was not significant $[F(1,4)=0.48, p=0.53]$. These analyses show that when the test rectangles did not cover the region of the luminance gradient of the adapting stimulus (the modified COC stimulus), the effect of luminance adaptation was significantly reduced. This suggests that the induced lightness on the adjacent surface area itself causes a significantly smaller luminance adaptation effect compared to the physical luminance stimulus, which has subjectively the same lightness (see the COC flanking test and Phys flanking test conditions).

\section{DISCUSSION}

\section{PRINCIPLE FINDINGS}

Using visual masking and interocular suppression paradigm, we found that the online percept of the COC effect nearly vanishes when the COC edge is rendered invisible. The result of BM experiments, in which the subjective percept of the COC edge was suppressed by temporally adjacent stimulus, is a novel finding, but it is unclear whether the COC edge was processed, since the COC and the mask stimuli were presented at the same retinal location. On the other hand, it was guaranteed that the retinal inputs were preserved at the stage of monocular processing in the CFS experiment, since the COC and the Mondrian stimulus were presented to different eyes. The results of the CFS experiment agree with a previous observation (see Supplemental Data of Boyaci et al., 2007), and in our experiment the effect of presentation duration of the COC stimulus tested systematically. In addition, the results are further supported by a recent report that the binocular rivalry suppresses the COC effect (see Shevell et al., 2011).

More importantly, the results of the adaptation experiments performed to test the processing stage of the COC edge under the CFS demonstrated that the neural processing of the COC edge was intact, presumably at the stage of monocular processing, even when the COC edge was invisible. This suggests that the neural processing of the COC edge at the early stage is not sufficient for generating lightness induction in the COC effect, and rather indicates that subjective awareness of the COC edge is crucial. Additionally, a subsidiary adaptation experiment demonstrated that the induced lightness of the adjacent surface showed a weaker adaptation effect than that caused by a subjectively equivalent physical stimulus, which also indicates the involvement of higher processing for lightness induction in the COC effect.

\section{MECHANISMS OF LIGHTNESS INDUCTION}

It is known that simple lightness induction effects, such as simultaneous contrast effects and Mach bands, can be explained by spatial filtering, say, lateral inhibition among neurons in the retina. Although the COC effect differs from such effects in its region of induction (the COC edge affects the perception of entire large areas) and its direction of induction (the region adjacent to the lighter part of the COC edge appears lighter, the opposite of the usual contrast effects), one might consider that the mechanism of the COC effect is similar to that of low-level-filtering effects (Békésy, 1972; Heggelund and Krekling, 1976). It is likely that such simple lateral inhibitions may occur at the earliest processing stage on the retina, but recent studies indicated the involvement of cortical processing (e.g., Perna et al., 2005; Boyaci et al., 2007; Hung et al., 2007), and our results further support the involvement of higher processing related to subjective awareness in the COC effect.

The activities related to the lightness induction are found in the broader areas of visual cortex. Recent brain imaging and physiological studies have raised the possibility that the neural mechanisms underlying lightness induction could be operated at the earliest stages of cortical visual processing in V1 or V2 (Rossi et al., 1996; Rossi and Paradiso, 1999; Hung et al., 2001, 2007; Kinoshita and Komatsu, 2001; MacEvoy and Paradiso, 2001; Roe et al., 2005). Also, several psychophysical studies implied that the surface lightness is encoded by the cortical filling-in process (Grossberg and Todorovic, 1988; Neumann et al., 2001) or banks of spatial frequency filters (Dakin and Bex, 2003; Perna and Morrone, 2007). Furthermore, another brain imaging study (Perna et al., 2005) indicated that the COC effect activates higher cortical areas, such as LO, which has been considered to be responsible for amodal completion (Sasaki and Watanabe, 2004). Although the brain areas responsible for the neural processing of the lightness induction are still unspecified, our results suggest that conscious processing of the COC edge, not automatic or unconscious processing, is required in order to trigger the lightness induction into the adjacent surfaces in the COC effect (see also Harris et al., 2011 for simultaneous contrast effect and Kanizsa illusion).

The series of our experimental results lead us to hypothesize that two mechanisms are responsible for the COC effect: a feedback mechanism in a hierarchical system, which is associated with subjective awareness of the COC edge, and a mechanism for surface lightness, which may rely on lateral connectivity and be activated by the feedback signal (Lamme and Roelfsema, 2000; Lamme, 2006; see also Bair et al., 2003 for a physiological study that showed a fast feedback mechanism and slow low-level mechanism relying on lateral connectivity in the primary visual cortex). It might be speculated that the feedback mechanism was disrupted by the BM and CFS stimuli and that the second mechanism for lightness induction was not activated, resulting in a failure of observation of the COC effect. Our hypothesis remains highly speculative at the moment, but it might also explain the results of the subsidiary adaptation experiment that the negative aftereffect caused by the illusory lightness on the surfaces was very 
limited and much smaller compared to conditions where observers were adapted with luminance rectangles that mimicked the illusory COC percept (see Figure 4E). Under the hypothesis, no input was given to the first feedback mechanism in the test phase, since the test stimulus did not cover the area of the luminance gradient of the modified COC stimulus, and the second mechanism responsible for the lightness induction was not activated. In summary, our results imply that the COC effect is an outcome of synthetic processing including feedback mechanisms, not solely an outcome of either early-stage processing of lateral interactions or higher-stage processing associated awareness.

The COC effect has been explained by another line of view, which is based on the empirical notion that the percept is determined by a statistical consequence of an accumulation of past experience rather than a veridical representation of the objects in the environment (Purves et al., 1999, 2004). The results of the BM and CFS experiments might be concomitant with the empirical view, assuming that the invisible COC edge could not be embedded in the empirical strategy as the contextual information to infer lightness of the surface area. On the other hand, the interpretation of the adaptation results with the empirical view seems to be more complicated. Although, in the empirical view, the visible contents could be considered as an essential quality for the inference of the global scene, previous studies suggested that invisible contents could affect perception of subsequent visual features (Lehmkuhle and Fox, 1975; O'shea and Crassini, 1981; Blake et al., 2006; Maruya et al., 2008). Our results of the adaptation experiments

\section{REFERENCES}

Adelson, E. H. (1993). Perceptual organization and the judgment of brightness. Science 262, 2042-2044.

Bair, W., Cavanaugh, J. R., and Movshon, J. A. (2003). Time course and time-distance relationships for surround suppression in macaque V1 neurons. J. Neurosci. 23, 7690-7701.

Békésy, G. V. (1972). Compensation method to measure the contrast produced by contours. J. Opt. Soc. Am. 64, 1247-1251.

Blake, R., and Camisa, J. (1979). On the inhibitory nature of binocular rivalry suppression. J. Exp. Psychol. Hum. Percept. Perform. 5, 315-323.

Blake, R., Tadin, D., Sobel, K. V., Raissian, T. A., and Chong, S. C. (2006). Strength of early visual adaptation depends on visual awareness. Proc. Natl. Acad. Sci. U.S.A. 103, 4783-4788.

Boyaci, H., Fang, F., Murray, S. O., and Kersten, D. (2007). Responses to lightness variations in early human visual cortex. Curr. Biol. 17, 989-993.

Breitmeyer, B. G., and Ganz, L. (1976). Implications of sustained and transient channels for theories of visual pattern masking, saccadic suppres- sion, and information processing. Psychol. Rev. 83, 1-36.

Breitmeyer, B. G., and Ogmen, H. (2000). Recent models and findings in backward visual masking: a comparison, review, and update. Percept. Psychophys. 62, 1572-1595.

Cornsweet, T. N. (1970). Visual Perception. New York: Academic Press. of Psychology: A Selection of Papers, Essays and Other Writings. New York: Cambridge University Press.

Dakin, S., and Bex, P. (2003). Natural image statistics mediate brightness 'filling-in'. Proc. Biol. Sci. 270, 2341-2348.

Davey, M. P., Maddess, T., and Srinivasan, M. V. (1998). The spatiotemporal properties of the CraikO'Brien-Cornsweet effect are consistent with 'filling-in'. Vision Res. 38, 2037-2046.

De Valois, R. L., Webster, M. A., De Valois, K. K., and Lingelbach, B. (1986). Temporal properties of brightness and color induction. Vision Res. 26, 887-897.

Devinck, F., Hansen, T., and Gegenfurtner, K. R. (2007). Temporal properties of the chromatic and achromatic craik-O'BrienCornsweet effect. Vision Res. 47, 3385-3393.
Craik, K. J. W. (1966). The Nature

showed that prolonged viewing of the invisible COC edge influenced subsequent lightness perception of the same retinal location (COC + CFS condition), but not that of adjacent flanking surface areas (COC + CFS flanking test condition). This suggests that invisible features could affect effective strength of subsequent inputs within spatially restricted areas, but could not play a role to infer subsequent global scenes in the lightness perception.

\section{CONCLUSION}

To investigate the underlying mechanisms of the COC effect, we examined whether the subjective awareness of the COC edge is necessary for the generation of the COC effect. We used visual $\mathrm{BM}$ and CFS to manipulate the visibility of the COC edge while obtaining subjective reports about the percepts and aftereffects of adaptation. Psychophysical results showed that the online percept of COC effect nearly vanishes in conditions where the COC edge is rendered invisible. On the other hand, the results of adaptation experiments showed that the COC edge is still processed at the stage of monocular processing. These results suggest that conscious processing of the COC edge is crucial for generating the COC effect and that automatic processing of the COC edge, presumably at the early stage of visual processing, is not sufficient.

\section{ACKNOWLEDGMENTS}

This research was supported by a Grant in Aid for Scientific Research (21530780), a Grant in Academic Frontier Promotion Project provided by MEXT.

Fink, W. H. (1938). The dominant eye: its clinical significance. Arch. Ophthalmol. 4, 555-582.

Gilchrist, A. L. (1977). Perceived lightness depends on perceived spatial arrangement. Science 95, 185-187.

Grossberg, S., and Todorovic, D. (1988). Neural dynamics of 1-D and 2$\mathrm{D}$ brightness perception:a unified model of classical and recent phenomena. Percept. Psychophys. 43 241-277.

Harris, J. J., Schwarzkopf, D. S., Song, C., Behrami, B., and Rees, G. (2011). Contextual illusions reveal the limit of unconscious visual processing. Psychol. Sci. 22, 399-405.

Heggelund, P., and Krekling, S. (1976). Edge-dependent lightness distributions at different adaptation levels. Vision Res. 16, 493-496.

Hung, C. P., Ramsden, B. M., Chen, L. M., and Roe, A. W. (2001). Building surfaces from borders in areas 17 and 18 of the cat. Vision Res. 41, 1389-1407.

Hung, C. P., Ramsden, B. M., and Roe, A. W. (2007). A functional circuitry for edge-induced brightness perception. Nat. Neurosci. 10, 1185-1190.

Kaufman, L. (1963). On the spread of suppression and binocular rivalry. Vision Res. 3, 401-415.
Kinoshita, M., and Komatsu, H. (2001). Neural representation of the luminance and brightness of a uniform surface in the macaque primary visual cortex. J. Neurophysiol. 86, 2559-2570.

Knill, D. C., and Kersten, D. (1991). Apparent surface curvature affects lightness perception. Nature 351, 228-230.

Komatsu, H. (2006). The neural mechanisms of perceptual filling-in. Nat. Rev. Neurosci. 7, 220-231.

Lamme, V. A. (2006). Towards a true neural stance on consciousness. Trends Cogn. Sci. (Regul. Ed.) 10, 494-501.

Lamme, V. A., and Roelfsema, P. R. (2000). The distinct modes of vision offered by feedforward, and recurrent processing. Trends Neurosci. 23, 571-579.

Lehmkuhle, S. W., and Fox, R. (1975). Effect of binocular rivalry suppression on the motion aftereffect. Vision Res. 15, 855-859.

Liu, L., and Schor, C. M. (1994) The spatial properties of binocular suppression zone. Vision Res. 34 937-947.

MacEvoy, S. P., and Paradiso, M. A. ((2001)). Lightness constancy in primary visual cortex. Proc. Natl. Acad. Sci. U.S.A. 98, 8827-8831. 
Maruya, K., and Blake, R. (2009). Spatial spread of interocular suppression is guided by stimulus configuration. Perception 38, 215-231.

Maruya, K., Hosokawa, K., Kusachi, E., Nishida, S., Tachibana, M., and Sato, T. (2010). A system for rapid development and easy sharing of accurate demonstrations for vision science. Conference Abstract: Neuroinformatics 2010. Front. Neurosci. doi: 10.3389/conf.fnins.2010.13.00093

Maruya, K., Watanabe, H., and Watanabe, M. (2008). Adaptation to invisible motion results in low-level but not high-level aftereffects. J. Vis. 8, 7.1-11.

Neumann, H., Pessoa, L., and Hansen, T. (2001). Visual filling-in for computing perceptual surface properties. Biol. Cybern. 85, 355-369.

O’Brien, V. (1958). Contour Perception, illusion and reality. J. Opt. Soc. Am. 48, 112-119.

O'shea, R. P., and Crassini, B. (1981). Interocular transfer of the motion after-effect is not reduced by binocular rivalry. Vision Res. 21, 801-804.

Paradiso, M. A., and Nakayama, K. (1991). Brightness perception and filling-in. Vision Res. 31, 1221-1236.

Perna, A., and Morrone, M. C. (2007). The lowest spatial frequency channel determines brightness perception. Vision Res. 47, 1282-1291.

Perna, A., Tosetti, M., Montanaro, D., and Morrone, M. C. (2005) Neuronal mechanisms for illusory brightness perception in humans. Neuron 47, 645-651.

Purves, D., Shimpi, A., and Lotto, R. B. (1999). An empirical explanation of the cornsweet effect. J. Neurosci. 19, 8542-8551.

Purves, D., Williams, S. M., Nundy, S., and Lotto, R. B. (2004). Perceiving the intensity of light. Psychol. Rev. 111, 142-158.

Rees, G., Kreiman, G., and Koch, C. (2002). Neural correlates of consciousness in humans. Nat. Rev. Neurosci. 3, 261-270.

Robinson, A. E., and de Sa, V. R. (2008). Brief presentations reveal the temporal dynamics of brightness induction and White's illusion. Vision Res. 48, 2370-2381.

Roe, A. W., Lu, H. D., and Hung, C. P. (2005). Cortical processing of a brightness illusion. Proc. Natl. Acad. Sci. U.S.A. 102, 3869-3874.

Rossi, A. F., and Paradiso, M. A. (1996). Temporal limits of brightness induction and mechanisms of brightness perception. Vision Res. 36, 1391-1398.
Rossi, A. F., and Paradiso, M. A. (1999). Neural correlates of perceived brightness in the retina, lateral geniculate nucleus, and striate cortex. J. Neurosci. 19 6145-6156.

Rossi, A. F., Rittenhouse, C. D., and Paradiso, M. A. (1996). The representation of brightness in primary visual cortex. Science 273, 1104-1107.

Sasaki, Y., and Watanabe, T. (2004). The primary visual cortex fills in color Proc. Natl. Acad. Sci. U.S.A. 101, 18251-18256.

Shevell, S., Allen, E., and Anstis, S. (2011). Binocular fusion unmasks rivalrous suppression of the CraikO'Brien-Cornsweet (COC) Illusion. Conference Abstract: Vision Sciences Society 11th Annual Meeting. J. Vis. 11,370 .

Tsuchiya, N., and Koch, C. (2005). Continuous flash suppression reduces negative afterimages. Nat. Neurosci. 8, 1096-1101.

von der Heydt, R., Friedman, H. S., and Zhou, H. (2003). "Searching for the neural mechanisms of color filling-in," in Filling-in: From Perceptual Completion to Cortical Reorganization, eds L. Pessoa and P. De Weerd (New York: Oxford University Press), 106-127.
Wachtler, T., and Wehrhahn, C. (1997). The Craik-O'Brien-Cornsweet illusion in colour: quantitative characterisation and comparison with luminance. Perception 26, 1423-1430.

Conflict of Interest Statement: The authors declare that the research was conducted in the absence of any commercial or financial relationships that could be construed as a potential conflict of interest.

Received: 21 July 2011; accepted: 13 October 2011; published online: 28 October 2011.

Citation: Masuda A, Watanabe J, Terao $M$, Watanabe $M$, Yagi $A$ and Maruya $K$ (2011) Awareness of central luminance edge is crucial for the Craik-O'BrienCornsweet effect. Front. Hum. Neurosci. 5:125. doi: 10.3389/fnhum.2011.00125 Copyright (c) 2011 Masuda, Watanabe, Terao, Watanabe, Yagi and Maruya. This is an open-access article subject to a nonexclusive license between the authors and Frontiers Media SA, which permits use, distribution and reproduction in other forums, provided the original authors and source are credited and other Frontiers conditions are complied with. 